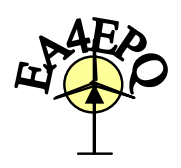

International Conference on Renewable Energies and Power Quality (ICREPQ'09)

Valencia (Spain), 15th to 17th April, 2009

\title{
An estimation and synchronization method based on a new modeling approach of power electrical signals
}

\author{
G. Fedele, C. Picardi and D. Sgrò \\ Department of Electronics, Computer and System Science \\ University of Calabria \\ Via Pietro Bucci 42C - 87036 Rende (Italy) \\ Phone number:+39 984 494719, e-mail: fedele@si.deis.unical.it, picardi@deis.unical.it, dsgro@deis.unical.it
}

\begin{abstract}
The authors have recently presented a new modeling approach, for electrical signals in power systems, which is based on the concept, derived from differential geometry, of the contact between a signal and an "osculating sinusoid". A difficulty in applying the approach lies in the calculation of the "osculating sinusoid" parameters, when the signal contains noise. In order to overcome this problem, the paper proposes a new computation algorithm for determining the "osculating sinusoid" parameters also in presence of noise on the signal. Accodingly, a new method providing a fast and accurate estimation of the frequency, phase and amplitude for noisy grid signals is developed. The good performance of the method is verified by some significant simulation results.
\end{abstract}

\section{Key words}

Distributed Generation, Frequency Detection, Modeling, Grid-connected inverter, Numerical Differentiation.

\section{Introduction}

Distributed generation (DG) systems are becoming more common as a result of the increased demand for electricity and the requirement to reduce the impact on the environment from traditional fossil and nuclear sources of power production.

DG systems are relatively small and many of them make use of renewable energy such as photovoltaic, fuel cell, microturbine or small wind power. They have a reverse power flow capability and operate in parallel with utility grid by means of intelligent power interfaces. The correct operation of these interfaces (very often grid-connected inverters) is assured by information about frequency, phase and amplitude of the utility voltage and current.

Moreover, an accurate and fast detection of the above quantities is also one of the most important issues regarding the development of power conditioning equipments such as Uninterrupted Power Systems and Series or Shunt Compensators (SC) [1],[2].

Thus, many estimation and synchronization methods, like suitable Phase Locked Loop (PLL) algorithms, have been recently developed and proposed to track the aforesaid information both in the steady state and during the transient periods [3]-[5].

On the other hand, especially considering the capacity of SCs and DG systems to operate services as power management or voltage control at distribution level, one can easily understand the importance of developing new approaches able to represent grid voltages and currents both in the steady state conditions as well as in the transient ones [6],[7].

For this reason, the authors have recently presented a new modeling approach for electrical signals in power systems [8]. The approach is based on the concept, derived from differential geometry, of the contact between a signal and an osculating sinusoid. A difficulty in its application lies in the calculation of the osculating sinusoid parameters, when the signal contains noise.

In order to overcome this problem, this paper proposes a new computation algorithm for determining the above parameters also in presence of noise on signal. Accordingly, a new method providing a fast and accurate estimation of the frequency, phase and amplitude for noisy grid signals is developed. The algorithm is based on the derivatives estimation of noisy time signals approaches, recently presented in literature [9]-[11].

The paper is organized as follows: the foundations of the modeling approach of power electrical signals are illustrated in the next section. In section 3, the new computation algorithm for determining the parameters associated with the approach, also in presence of noise on the signals, is described in detail. Then, simulation results about the direct application of the modeling approach as estimation and synchronization method in some significant situations are given in section 4 .

\section{New Modeling Approach of Signals}

The modeling approach already proposed in [8] is based on the concept of the contact between two curves on $R^{2}$ in differential geometry. In particular, two curves on $R^{2}$ described by the real functions $y=f(x)$ and $y=g(x)$, continuous and with continuous derivatives up to an order of interest, have a $n$-th order contact at point $P$ of 
abscissa $x_{0}$ if the two functions and their derivatives up the order $n$ have the same values, whereas the derivatives of order $n+1$ are different i.e.:

$$
\begin{aligned}
& f\left(x_{0}\right)=g\left(x_{0}\right) ; \quad f^{(1)}\left(x_{0}\right)=g^{(1)}\left(x_{0}\right) ; \ldots \ldots ; \\
& f^{(n)}\left(x_{0}\right)=g^{(n)}\left(x_{0}\right) ; \quad f^{(n+1)}\left(x_{0}\right) \neq g^{(n+1)}\left(x_{0}\right) .
\end{aligned}
$$

A zero order contact means that the curves have a simple crossing at $P$, a first order contact corresponds to two curves tangent at $P$ and two curves having a second order contact are said osculating. So, for example the osculating circle of a curve at a point is just a circle having a second order contact (at least) with the curve at that point. Indeed, the three conditions obtained by (1) and specified with $n=2$, give three equations allowing to determine, for each $x_{0}$, the radius and the coordinates of the center of a unique circle, provided that the second derivative is not zero.

In a similar way, for a generic electrical signal $y(t)$ it is possible to define as "osculating sinusoid" of $y(t)$ at instant $t_{0}$, the signal $v(t)$ given by:

$$
v(t)=V(t) \sin [\theta(t)] \quad \text { with } \quad \theta(t)=\omega(t) t+\varphi(t)
$$

and meeting the following conditions:

$v\left(t_{0}\right)=y\left(t_{0}\right), \quad v^{(1)}\left(t_{0}\right)=y^{(1)}\left(t_{0}\right), \quad v^{(2)}\left(t_{0}\right)=y^{(2)}\left(t_{0}\right)$.

Denoting $\quad V\left(t_{0}\right)=V_{0}, \quad \omega\left(t_{0}\right)=\omega_{0}, \varphi\left(t_{0}\right)=\varphi_{0} \quad$ and then $\theta\left(t_{0}\right)=\omega_{0} t+\varphi_{0}$, the above conditions provide the following three equations:

$$
\begin{aligned}
& V_{0} \sin \left[\theta\left(t_{0}\right)\right]=y\left(t_{0}\right), \\
& \omega_{0} V_{0} \cos \left[\theta\left(t_{0}\right)\right]=y^{(1)}\left(t_{0}\right), \\
& \omega_{0}^{2} V_{0} \sin \left[\theta\left(t_{0}\right)\right]=-y^{(2)}\left(t_{0}\right) .
\end{aligned}
$$

Through simple manipulations, the following solutions can be obtained:

$$
\begin{aligned}
& \omega_{0}=\sqrt{-y^{(2)}\left(t_{0}\right) / y\left(t_{0}\right)}, \\
& \theta\left(t_{0}\right)=\operatorname{arctg}\left[\omega_{0} y\left(t_{0}\right) / y^{(1)}\left(t_{0}\right)\right], \\
& V_{0}=\sqrt{\left[y\left(t_{0}\right)\right]^{2}+\left[y^{(1)}\left(t_{0}\right) / \omega_{0}\right]^{2}} .
\end{aligned}
$$

It is easy to observe that the angular frequency given by (6) is not real when the following condition is verified:

$$
y^{(2)}\left(t_{0}\right) / y\left(t_{0}\right)>0 .
$$

In order to define, however, an "osculating sinusoid", we substitute (5) with the following equation:

$$
\omega_{0}^{2} V_{0} \sin \left[\theta\left(t_{0}\right)\right]=\operatorname{sgn}\left[\frac{y^{(2)}\left(t_{0}\right)}{y\left(t_{0}\right)}\right] y^{(2)}\left(t_{0}\right)
$$

and so the angular frequency is determined by:

$$
\omega_{0}=\sqrt{\left|y^{(2)}\left(t_{0}\right) / y\left(t_{0}\right)\right|} .
$$

In this way, it is always possible to define the signal $v(t)$, given in (2), by which the electrical signal $y(t)$ can be locally approximated. Clearly, this signal, still called "osculating sinusoid", actually has not a second order contact with $y(t)$ when condition (9) occurs.

In order to better clarify the peculiarities of the modeling approach, we consider to apply it to a generic signal, for example to $y(t)=t^{3} e^{-t}$. By using (11), (7) and (8), it is possible to determine the "osculating sinusoids" practically for every instant. Fig. 1 shows the signal to be modelled (in red) and the osculating sinusoids (in blue and green) in two different instants.

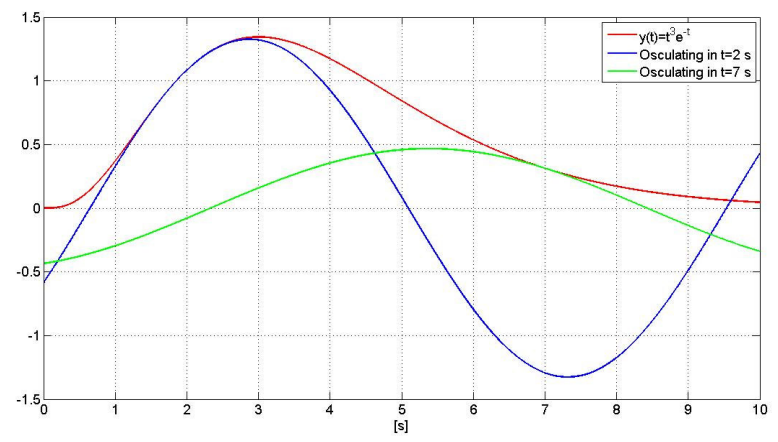

Fig. 1. "Osculating sinusoids" of $y(t)=t^{3} e^{-t}$ for $t=2 \mathrm{~s}$ (in blue) and for $t=7 \mathrm{~s}$ (in green)

It is important to note that, if $y(t)$ is just a sinusoidal signal, then the "osculating sinusoid" $v(t)$ is clearly the same at the various instants with amplitude, angular frequency and phase equal to those of $y(t)$. On the other hand, it is worth observing that the power electrical signals, for which it is proposed to apply the modeling approach, are generally characterized by waveforms, that converge to sinusoidal signals of grid frequency.

\section{A. Calculation of the Osculating Sinusoid's Parameters}

As described in [8], because in some istants the quotient included in (11) is ill defined due to an indetermination of the form $0 / 0$, it can be convenient to use a computation algorithm based on the knowledge also of the third order derivative of the signal. Accordingly, the parameter $\theta\left(t_{0}\right)$ can be calculated by:

$$
\theta\left(t_{0}\right)=\operatorname{arctg}\left[\sqrt{\frac{y^{(3)}\left(t_{0}\right)}{y^{(1)}\left(t_{0}\right)}} \frac{y\left(t_{0}\right)}{y^{(1)}\left(t_{0}\right)}\right]
$$

and then $\omega_{0}$ can be obtained by:

$\omega_{0}=\sqrt[4]{\left[\frac{y^{(2)}\left(t_{0}\right)}{y\left(t_{0}\right)} \operatorname{sen}\left[\theta\left(t_{0}\right)\right]\right]^{2}+\left[\frac{y^{(3)}\left(t_{0}\right)}{y^{(1)}\left(t_{0}\right)} \cos \left[\theta\left(t_{0}\right)\right]\right]^{2}}$. 
The amplitude $V_{0}$ is still determined by means of (8). Then, to apply this computation algorithm, it is necessary to have a practically instantaneous estimation of signal $y(t)$ and of its derivatives up to third order at instant $t_{0}$. Different numerical algorithms able to give fast and sufficiently accurate estimations of the above quantities from the knowledge of few samples of signal $y(t)$ can be chosen [8].

\section{B. Applications of the Modeling Approach}

As known, a PLL structure first must provide the phase of the grid voltage so as to synchronize the inverter output current with the grid voltage; at the same time the structure also allows to monitor the grid voltage amplitude and angular frequency.

The direct application of the proposed modeling approach leads to an algorithm performing tasks comparable to those of a single-phase PLL for gridconnected inverters. Indeed, as confirmed by significant simulation results shown in [8], the modeling approach provides an algorithm able to give fast and accurate synchronization and reliable monitoring of the amplitude and frequency of the grid voltage.

The basic point of view of the approach, consisting in considering the signal $y(t)$ as a sinusoidal signal of angular frequency, phase and amplitude varying with respect to time, involves a very interesting result. Indeed, it allows to extend the same concepts used in steady state conditions to characterize the grid signals, also in the transient operation; then, new definitions of instantaneous active and reactive powers can be used to design new power flow control strategies in applications based on power electronics converters [7],[8].

\section{A New Algorithm for Noisy Signals}

A problem in applying the above modeling approach is actually due to the fact that the equations (8),(12) and (13) used to determine the parameters of the "osculating sinusoid", require the knowledge of the signal $y(t)$ and its derivatives up to third order for every instant of time. Indeed, it is known that the measure of an electrical signal contains at least the acquisition noise; therefore, even if suitable numerical differentiation algorithms are adopted, the calculated derivatives, especially those of higher order, are acceptable only for noise-free signals.

With the aim of overcoming this problem, this paper proposes a new computation algorithm, which takes into account the presence of noise on the signal. Consequently, this algorithm, applied to the modeling approach of the power electric signals, allows to obtain a method providing a fast and accurate estimation of the frequency, phase and amplitude for noisy signals.

This algorithm is divided into three steps, which provide the estimations of the angular frequency, angle phase and amplitude respectively for the "osculating sinusoid" at the generic instant $t_{0}$.

The algorithm is based on the derivatives estimation of noisy time signals approaches, recently presented in literature [9]-[11].

\section{A. Estimation of the Angular Frequency}

In this new context, the real noisy signal is:

$$
r(t)=y(t)+n(t)
$$

where $n(t)$ is an unstructured perturbation and $y(t)$ is the noise-free signal. To estimate the angular frequency $\omega_{0}$ of the "osculating sinusoid" at instant $t_{0}$, we assume to represent the signal $r(t)$ according to the model:

$$
r(t) \approx v(t)+n(t) \quad t_{0}-T \leq t \leq t_{0},
$$

where $v(t)$ is just the "osculating sinusoid" at $t_{0}$ i.e. $v(t)=V_{0} \sin \left(\omega_{0} t+\varphi_{0}\right)$ and $T$ indicates the size of sliding estimation window. This hypothesis can certainly be acceptable, if we use a quite short time window, nevertheless sufficient to obtain accurate estimations.

In order to simplify the description of the algorithm, we consider, for the moment, that (15) is valid for $t \geq 0$; this corresponds to asssume that the noise-free signal is actually sinuoidal with constant angular frequency to be estimated.

It is easily to observe that the signal $v(t)$ satisfies the following linear differential equation with constant coefficient:

$$
v^{(2)}(t)+\omega_{0}^{2} v(t)=0
$$

Translating this equation into operational domain, we get:

$$
s^{2} V(s)-s v(0)-v^{(1)}(0)+\omega_{0}^{2} V(s)=0
$$

Taking the second derivative with respect to $s$ permits to ignore the initial conditions:

$$
2 V(s)+4 s \frac{d V(s)}{d s}+\left(s^{2}+\omega_{0}^{2}\right) \frac{d^{2} V(s)}{d s^{2}}=0 .
$$

Then, dividing both sides by $s^{3}$ to avoid derivations with respect to time, we have:

$$
\frac{2}{s^{3}} V(s)+\frac{4}{s^{2}} \frac{d V(s)}{d s}+\left(\frac{1}{s}+\frac{1}{s^{3}} \omega_{0}^{2}\right) \frac{d^{2} V(s)}{d s^{2}}=0 .
$$

To estimate the parameter $\omega_{0}^{2}$, it needs to express (16) in time domain, using the classic rules of operation calculus. Recalling that the derivation $d^{k} / d s^{k}$ with respect to $s$ translates into the multiplication by $(-1)^{k} t^{k}$ and $1 / s^{k}$ is replaced by the iterated integral of order $k$, we obtain, thanks to Cauchy rule, the estimation of $\omega_{0}^{2}$ as the following time-function:

$$
\omega_{0 e}^{2}(t)=-2 \frac{\int_{0}^{t}\left[(t-\tau)^{2}-4(t-\tau) \tau+\tau^{2}\right] r(\tau) d \tau}{\int_{0}^{t}(t-\tau)^{2} \tau^{2} r(\tau) d \tau},
$$


where of course, the noisy observation $r(t)$ is considered in the two integrals. Let us note that (17) gives a timedomain representation with no derivatives but only integrations with respect to time and the unstructured noise is attenuated by the iterated time integrals, which are simple examples of low pass filters.

Specifying this result to the representation (15) and taking into account closely the definition given in the previous section, we obtain for the estimation of the angular frequency $\omega_{0 e}$ of the "osculating sinusoid" at instant $t_{0}$, the following formula:

$$
\omega_{0 e}^{2}=2\left|\frac{\int_{t_{0}-T}^{t_{0}}\left[(T-\tau)^{2}-4(T-\tau) \tau+\tau^{2}\right] r(\tau) d \tau}{\int_{t_{0}-T}^{t_{0}}(T-\tau)^{2} \tau^{2} r(\tau) d \tau}\right| .
$$

\section{B. Estimation of the Phase Angle}

According to (7), it is easy to obtain the following estimation $\theta_{e}\left(t_{0}\right)$ of the phase angle $\theta\left(t_{0}\right)$ :

$$
\theta_{e}\left(t_{0}\right)=\operatorname{arctg}\left[\omega_{0 e} y_{e}\left(t_{0}\right) / y_{e}^{(1)}\left(t_{0}\right)\right]
$$

where $y_{e}\left(t_{0}\right)$ and $y_{e}^{(1)}\left(t_{0}\right)$ denote the estimations of the noise-free signal and its first derivative respectively at instant $t_{0}$. Therefore, to obtain the estimation $\theta_{e}\left(t_{0}\right)$ it is necessary to extract from the samples of the noisy signal $r(t)$ in the interval $\left[t_{0}-T, t_{0}\right]$, the estimations of the angular frequency $\omega_{0 e}$, the noise-free signal and its first derivative.

The approach, adpoted to calculate these quantities, is based on the assumption that the noise-free signal $y(t)$ can be locally approximated in interval $\left[t_{0}-T, t_{0}\right]$ by the truncated Taylor expansion. However, for the sake of simplicity we first consider to represent $y(t)$ in the interval $[0, T]$ by the following polynomial function:

$$
y(t) \approx y(0)+\sum_{h=1}^{N} y^{(h)}(0) \frac{t^{h}}{h !},
$$

where $N$ is the approximation degree.

In order to obtain a quite accurate estimation of the first derivative of the noise-free signal, a degree $N=3$ has been chosen in (20) i.e. the following expansion has been considered:

$$
y(t) \approx y(0)+y^{(1)}(0) t+y^{(2)}(0) \frac{t^{2}}{2}+y^{(3)}(0) \frac{t^{3}}{6} .
$$

Translating this equation into operational domain, the previous equation is written as:

$$
Y(s) \approx \frac{y(0)}{s}+\frac{y^{(1)}(0)}{s^{2}}+\frac{y^{(2)}(0)}{s^{3}}+\frac{y^{(3)}(0)}{s^{4}} .
$$

Multiplying both sides of the equality by $s^{4}$ and taking the second derivative with respect to $s$, we have:

$$
12 s^{2} Y(s)+8 s^{3} \frac{d Y(s)}{d s}+s^{4} \frac{d^{2} Y(s)}{d s^{2}}=6 s y(0)+2 y^{(1)}(0) .
$$

Dividing by $s$, taking the derivative with respect to $s$, again dividing by $s^{4}$ and finally using the correspondence rules from operational domain to time domain and the Cauchy rule, the estimation of the first derivative $y_{e}^{(1)}(0)$ is given by:

$$
y_{e}^{(1)}(0)=-\frac{60}{T^{5}} \int_{0}^{T} p(\tau) r(\tau) d \tau
$$

with

$$
p(\tau)=2(T-\tau)^{3}-14(T-\tau)^{2} \tau+11(T-\tau) \tau^{2}-\tau^{3} .
$$

Because we are interested in obtaining the estimation $y_{e}^{(1)}\left(t_{0}\right)$, based on the samples of the noisy signal $r(t)$ in the interval $\left[t_{0}-T, t_{0}\right]$, it is easy to understand as its formula can be drawn from (21) and it has the following expression:

$$
y_{e}^{(1)}\left(t_{0}\right)=\frac{60}{T^{5}} \int_{t_{0}-T}^{T} p(\tau) r\left(t_{0}-\tau\right) d \tau \text {. }
$$

By using an algorithm similar to the previous one, with a degree $N=2$ in (20), the quantity $y_{e}\left(t_{0}\right)$ is given by:

$$
y_{e}\left(t_{0}\right)=\frac{3}{T^{3}} \int_{t_{0}-T}^{t_{0}} q(\tau) r\left(t_{0}-\tau\right) d \tau
$$

with

$$
q(\tau)=3(T-\tau)^{2}-6(T-\tau) \tau+\tau^{2}
$$

Once known the estimation $\theta_{e}\left(t_{0}\right)$, calculated by (19), immediately we get the estimation of phase $\varphi_{0}$.

\section{Estimation of the Amplitude}

The estimation of amplitude $V_{0 e}$ of the "osculating sinusoid" at instant $t_{0}$ is calculated by the equation:

$$
V_{0 e}=\sqrt{\left[y_{e}\left(t_{0}\right)\right]^{2}+\left[y_{e}^{(1)}\left(t_{0}\right) / \omega_{0 e}\right]^{2}}
$$

directly derived by (8).

\section{Simulation experiments}

The previous algorithm, applied to the modeling approach of the power electric signals, allows to obtain a method providing a fast and accurate estimation of the frequency, phase and amplitude for noisy grid signals. 
In order to explain the performances of this method both during stationary and transient conditions, this section reports some significant numerical experiments. In these experiments, the noise level, introduced in (14) and measured by the Signal to Noise Ratio in dB i.e.:

$$
S N R=10 \log _{10}\left(\frac{\sum\left|y\left(t_{i}\right)\right|^{2}}{\sum\left|n\left(t_{i}\right)\right|^{2}}\right)
$$

has been taken equal to $40 \mathrm{~dB}$; a sliding estimation window $T=2 \cdot 10^{-3} \mathrm{~s}$, with 2000 samples has been chosen. Suitable and simple numerical expedients have been used in order to avoid numerical errors in specific conditions. In particular, to avoid zero-crossing of the denominator in (18) an appropriate threshold has been adopted.

The first experiment allows to evaluate the performances of the angular frequency estimation obtained by (18). Actually, the quantity $f_{0 e}=\omega_{0 e} / 2 \pi$ has been taken into account; therefore, an abrupt change of the frequency of the signal, from 50 to $51 \mathrm{~Hz}$, has been considered.

Fig. 2 shows the frequency variation (in blue) and the estimation obtained by the proposed method (in red). As it is evident, after the step variation, the correct value of the frequency is estimated in less than an half of the signal period.

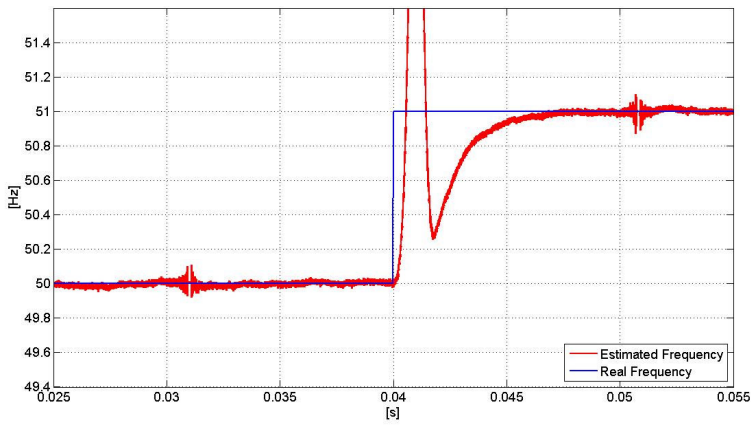

Fig. 2. Frequency estimation

In order to highlight the performance of the method as regards its ability to provide a good synchronization, the second experiment considers a ramp variation from $50 \mathrm{~Hz}$ to $60 \mathrm{~Hz}$ for the frequency of the signal.

Fig. 3 shows as the estimation of the angle phase $\theta(t)$ is accurate and there is always synchronization between the estimated angle phase (in red) and the real signal (in blue), also when the frequency is gradually changing. The third simulation experiment aims to highlight the ability of the proposed method to provide a correct estimation of all the signal parameters. Therefore, this experiment considers a variation of the signal amplitude which goes from 7 to 5 Volt and lasts about $90 \mathrm{~ms}$.

Fig. 4 shows again the comparison between real signal (in blue) and the estimated angle phase (in red) and also that between the real signal amplitude variation (in green) and its estimation (in black). The figure highlights the good estimation of the signal amplitude obtained also

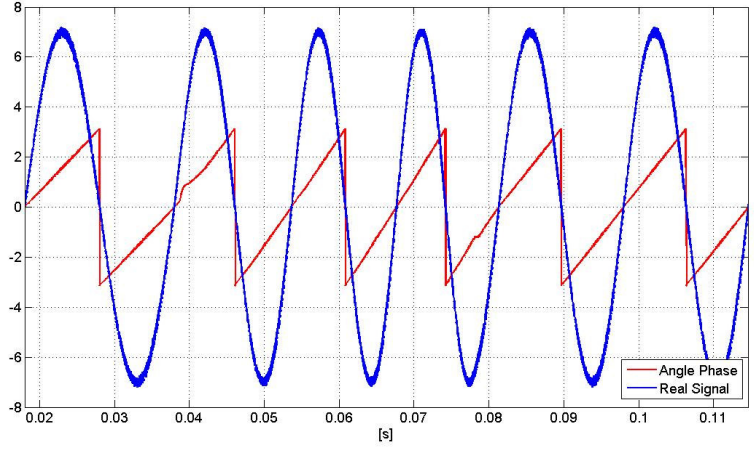

Fig. 3. Angle phase estimation

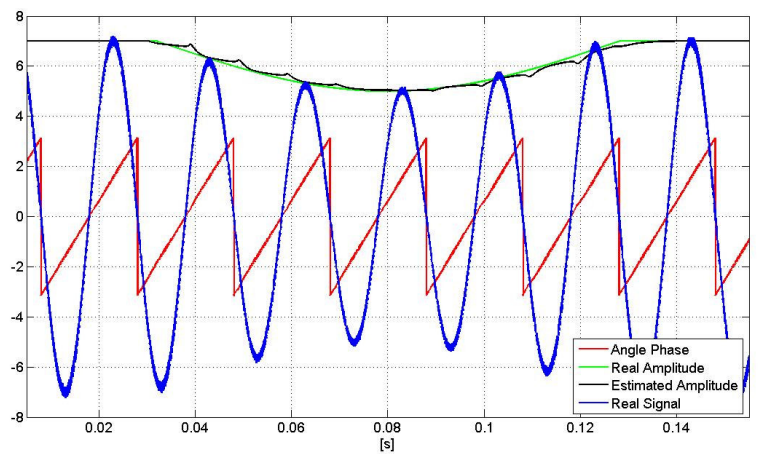

Fig. 4. Angle phase and amplitude estimations

during the transient condition considered, without losing synchronization between the estimation of the angle phase $\theta(t)$ and the real signal.

\section{Conclusion}

The paper started from a new modeling approach useful to represent power electrical signal. This approach is based on the definition of an "osculating sinusoid", by which the electrical signal can be locally approximated. A difficulty in its application lies in the calculation of the parameters of the "osculating sinusoid" as angular frequency, angle phase and amplitude, when the signal contains noise.

Therefore, this paper developed a new computational algorithm for determining the parameters of the "osculating sinusoid" for noisy signals. This algorithm was obtained by the derivatives estimations of noisy time signals approaches, recently presented in literature.

Then, this algorithm, applied to the modeling approach of signals, was used as method providing a fast and accurate estimation of the frequency, phase and amplitude for noisy grid signals.

Moreover, considering the capability of the modeling approach to extend concepts as frequency, phase angle and amplitude during the transient, the main advantage of the proposed method consists in the ability to provide very fast estimations in steady state conditions as well in transient ones. This is very useful in applications as DG systems or Power Conditioner equipments connected to the utility grid especially at distribution level. 


\section{References}

[1] P. Garcìa-Gonzàlez and A. Garcìa-Cerrada, "Control System for a PWM-Based STATCOM", IEEE Trans. On Power Delivery, Vol.15, No.4, 2000, pp.1252-1257.

[2] F. Blaabjerg, Z. Chen and S. Kjaer, "Power electronics as efficient interface in dispersed power generation systems", IEEE Trans. on Power Electronics, Vol. 19, No. 5, 2004 pp. 1184-1194.

[3] L. N. Arruda, S. M. Silva and B. J. Cardoso Filho, " PLL Structures for Utility Connected Systems", Industry Applications Conference, 2001, pp. 2655-2660.

[4] M. Ciobotaru, R. Teodorescu and F. Blaabjerg, "A New Single-Phase PLL Structure Based on Second Order Generalized Integrator", IEEE 37th Power Electronics Specialists Conference, June 2006 pp. 1-6.

[5] L. Coluccio, A. Eisinberg, G. Fedele, C. Picardi and D. Sgrò, "Modulating functions method plus SOGI scheme for signal tracking", IEEE International Symposium on Industrial Electronics, Cambridge (United Kingdom), June-July 2008, pp. 854-859 .

[6] H. Akagi, E. H. Watanabe and M. Aredes, "Instantaneous Power Theory and Applications to Power Conditioning", IEEE Press, Wiley-Interscience, 2007.
[7] D. Menniti, C. Picardi, A. Pinnarelli and D. Sgrò, "Gridconnected inverters for alternative energy sources with a combined voltage and current control strategy", ICCEP Intern. Conference, Capri (Italy), May 2007, pp. 223-228.

[8] C. Picardi and D. Sgrò, "A New Modeling Approach of Electrical Signals for Power Systems", SPEEDAM Intern. Symposium, Ischia (Italy), June 2008, pp. 912-917.

[9] M. Mboup, C. Join and M. Fliess, “ A revised look at numerical differentiation with an application to nonlinear feedback control", Proc. Of the $15^{\text {th }}$ Mediterranean Conference on Control \& Automation, Athens (Greece) July 2007, T23-006.

[10] J. R. Trapero, H. Sira-Ramìrez and V. F. Batlle, "An algebraic frequency estimator for a biased and noisy sinusoidal signal", Signal Processing, Vol. 87, 2007, pp.1188-1201.

[11] S. Rezk, C. Join, S. El Asmi, M. Dogui and M.H. Bedoui, "Frequency Change-Point Detection in physiological Signals: an Algebraic Approach", Int. Journal on Science and Techniques of Automatic control \& computer engineering, 2008, pp.1-13. 